\title{
Effects of differential prior exposure on preschool children's subsequent choice of novel stimuli
}

\author{
R. C. ENDSLEY \\ UNIVERSITY OF GEORGIA
}

Four groups of 12 preschool children were pre-exposed to one of two sets of seven toys for $0,1,3$, or $5 \mathrm{~min}$. Each $S$ then received 10 trials on which he could choose one of the two sets to play with for $30 \mathrm{sec}$. All $36 \mathrm{Ss}$ in the three nonzero exposure groups chose the novel (not previously exposed) set on trial 1. Further, the number of novel set choices over the 10 trials was an increasing function of the amount of prior exposure to the other set.

Recent findings with young children have supported Berlyne's proposition (1960) that stimuli possessing "short-term" novelty elicit a variety of curiosity behaviors (Cantor \& Cantor, 1964; Mendel, 1965; Harris, 1965). The typical procedure in these child studies has been to expose $S$ to one set of stimuli but not to a second set prior to test presentations of each. The main purpose of the present study was to explore the effects of several amounts of prior exposure to one of two sets of stimuli on subsequent tendencies to choose the previously nonexposed set. Specifically, it was predicted that with an increase in the amount of prior exposure to one of two sets of toys, over a series of 10 choice trials there would be both an increase in the number of choices of the novel (not previously exposed) set and a decrease in the number of alternations between the two sets. These two predictions were based on Berlyne's proposition that organisms tend to approach stimuli as a function of their novelty, and on the assumption that both the number of novel stimulus set choices and the number of alternations between the two sets (Berlyne, 1960; Dember, 1961) reflect S's tendency to maintain commerce with novel stimuli.

\section{Method}

The Ss were 24 boys and 24 girls from the University of South Dakota Nursery School. The mean age of the 48 children was 4.4 years, with a range from 3.3 to 5.6 years.

The stimulus materials were 14 toys purchased at a local dime store. None of the purchases exceeded 25 cents. The 14 toys were arranged into seven pairs according to the author's judgment that the pair members were more like each other on some physical and/or conceptual basis than they were like any of the remaining toys. One member of each pair was then randomly assigned to Toy Set A, the remaining member to Toy Set B. A verbal description of the toys in each of the seven pairs follows, with the first and second members of each pair belonging to
Set A and B, respectively: (1) black rubber knife, red plastic gun; (2) brown plastic dog, blue plastic mouse; (3) rubber doll, doll in high chair; (4) wooden rolling pin, girl's plastic watch; (5) red metal car, red metal tractor; (6) wood and metal zylophone, green plastic whistle; (7) blue plastic airplane, blue plastic telephone.

Each of the toy sets were arranged in a circular pattern on the two ends of a child-size table, with the ends on which each set was placed randomly determined for each $\mathrm{S}$. The two toy sets were separated by a wooden panel which was vertically positioned in the center of the table. A child-size chair was placed $6 \mathrm{ft}$. from the table. Cloth covers were placed over each toy set.

Upon entering the room, $S$ was told that he would have an opportunity to play with some toys. For each $S$ in the three groups receiving a non-zero prior exposure (see subsequent description of the design), E lifted the cover from one of the toy sets and invited $S$ to approach the table and play with the exposed toys. At the end of the prior exposure period the exposed set was again covered, and $S$ was returned to his chair. Ten choice trials followed. On each of the choice trials $\mathrm{E}$ simultaneously uncovered both sets and asked $\mathrm{S}$, "Which set of toys would you like to play with?" When $S$ made his choice, the other set of toys was covered again. Following his choice, $\mathrm{S}$ was allowed to play with the chosen toy set for 30 sec. At the end of the 30-sec, interval, E covered the exposed set and asked $S$ to return to his chair.

Six boys and six girls were randomly assigned to each of the four prior exposure groups: 0 min. exposure-G0; $1 \mathrm{~min}$. exposure-G1; $3 \mathrm{~min}$. exposureG3; and $5 \mathrm{~min}$. exposure-G5. Set A toys were designated as the prior exposure set for a random half of the boys and the girls in each prior exposure group; Set B was designated as the prior exposure set for the remaining $S s$ in each group. A novel set choice was defined as a selection of the toy set not assigned for prior exposure. An alternation was defined as choice of the set not selected by $\mathrm{S}$ on the preceding trial.

\section{Results and Discussion}

Figure 1 presents the mean number of novel set choices for Ss in each of the four prior exposure groups. Inspection of Fig. 1 indicates, as predicted, that the number of novel set choices was an increasing function of the amount of prior exposure. This 


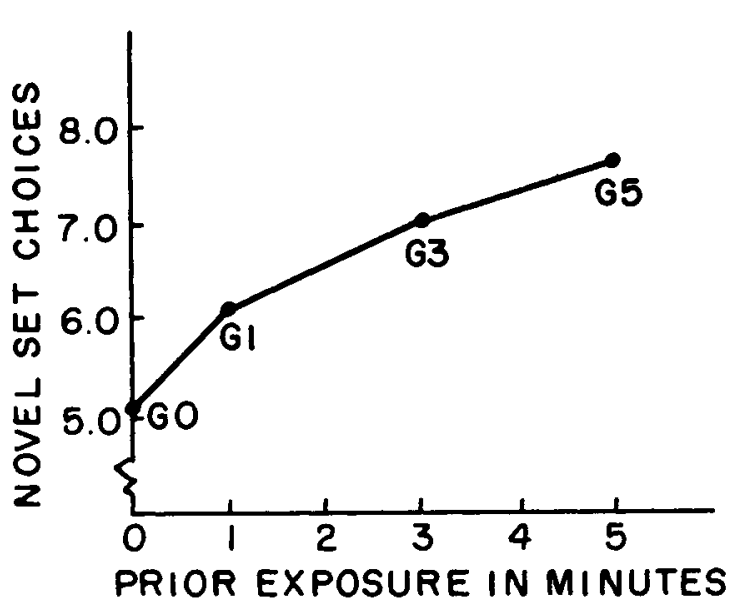

Fig. 1. Mean number of novel set choices made by Ss in Go, G1, G3, and G5.

conclusion was supported by the results of an analysis of variance, which revealed the effects of prior exposure to be significant ( $F=26.62, d f=3 / 44, p<.001$ ).

The shape of the curve in Fig. 1 also suggests that increasing prior exposure to one set did not result in a linear increase in the number of novel set choices that would have been expected if Ss were simply choosing the novel set a sufficient number of times to equate the total time spent with each set. According to an "equalization" hypothesis, for example, all Ss in G5 should have made 10 choices of the novel set in order to have accumulated $5 \mathrm{~min}$. (10 trials times 30 sec.) of contact with that set to compensate for the $5 \mathrm{~min}$. of play with the pre-exposed set. Similarly, Ss in G3, G1, and G0 should have chosen the novel set 8,6 , and 5 times, respectively. In general, the differences between the obtained novel set choice means and the means generated by an equalization hypothesis increased with increased prior exposure, with Ss in G3 and G5 making significantly fewer choices than "predicted."

Glanzer's stimulus satiation theory (1953) would seem to be able to account for the increasing divergence between the amount of contact with one set and the subsequent amount of contact with the other set during choice trials. The theory predicts that the greater the satiation to a set of stimuli, the greater the dissipation of satiation during subsequent moments when $S$ is not perceiving the stimuli. Thus, during the 10 intertrial intervals in the present study when both toy sets were covered, the amount of satiation dissipation to the pre-exposed set relative to the novel set (and hence, the amount of reduction in the difference in approach tendencies to the two sets) should have been greatest in G5, next greatest in G3, etc.

The shape of the curve in Fig. 1 might also be explained by appealing to the increased operation of "ceiling effects" with increased prior exposure.
That is, assuming the difference between S's tendencies to approach the novel set and the pre-exposed set varies by chance from moment to moment (Spence, 1956), then as S's net tendency to approach the novel set reaches the strength required to produce 10 novel set choices in 10 trials, momentary chance increases in that tendency would be less likely to offset momentary chance decreases in that tendency.

The mean number of alternations between the two sets over 10 trials for G0, G1, G3, and G5 were $5.8,6.3,4.9$, and 3.1 , respectively. While, as predicted, these results suggest an inverse relationship between prior exposure and alternation, results of an analysis of variance indicated that the effect of prior exposure failed to reach an acceptable (5\%) level of significance $(F=2.61, d f=3 / 44, p<.10)$.

A Chi square analysis was also performed on the number of Ss in G0 and in the combination of G1, G3, and G5 who selected the novel set on choice Trial 1. All 36 Ss in the G1-G3-G5 combination chose the novel set on Trial 1, while eight of the 12 G0 Ss chose the arbitrarily designated "novel" set. (The fact that eight of the $12 \mathrm{Ss}$ in G0 chose the "novel" set presumably represents a chance fluctuation from an expected value of six, since neither set had been pre-exposed.) A 2 by 2 Chi square analysis of the Trial 1 data was significant $\left(x^{2}=9.09, d f=1, p<.01\right)$, supporting the conclusion that prior exposure to one toy set for 1 to $5 \mathrm{~min}$. greatly facilitates choice of the other set on Trial 1. This finding is consonant with those of Harris (1965) and Mendel (1965) who found that on the first choice trial nearly all of their preschool Ss chose the toy or toy sets not previously exposed.

In conclusion, firm evidence with young children was obtained to indicate that increasing the relative degree of short-term novelty possessed by one of two toy sets increases the number of subsequent choices of that set. The effects of variations in the degree of short-term novelty on alternation behavior are less clear. However, the suggestive trend warrants further research on the role played by stimulus novelty in determining alternation in two-choice tasks.

\section{References}

Berlyne, D. E. Conflict, arousal, and cutiosity. New York: McGrawHill, 1960.

Cantor, Joan H., \& Cantor, G. N. Children's observing behavior as related to amount and recency of stimulus familiarization. $J$. exp. child Psychol., 1964, 1, 241-247.

Dember, W. N. Alternation behavior. In D. W. Fiske \& S. R. Maddi (Eds.), Functions of varied experience. Homewood: Dorsey, 1961. Glanzer, M. Stimulus satiation: An explanation of spontaneous alternation and related phenomena. Psychol. Rev., 1953, 60, 257-268.

Harris, $\mathbf{L}$. The effects of relative novelty on children's choice behavior. J. exp. child Psychol., 1965, 2, 297-305.

Mendel, Gisela. Children's preferences for differing degrees of novelty. Child Develpm., 1965, 36, 453-465.

Spence, K. W. Bcharior theory and conditioning. New Haven: Yale University Press, 1956. 